\title{
Hypertrophic Osteoarthropathy in Nasopharyngeal Carcinomas: A Rare Differential
}

\author{
${ }^{1}$ Satish K Srinivas, ${ }^{2}$ Arun Kumar, ${ }^{3} \mathrm{~A}$ Vasanthan, ${ }^{4}$ Divyaambica CV, ${ }^{5}$ Sathasiva Subramanium S \\ ${ }^{6}$ Majhi U, ${ }^{7}$ R Krishnakumar, ${ }^{8}$ Arvind Krishnamurthy \\ ${ }^{1}$ Assistant Professor, Department of Radiation Oncology, Cancer Institute (WIA), Adayar, Chennai, Tamil Nadu, India \\ ${ }^{2}$ Postgraduate Student, Department of Radiation Oncology, Cancer Institute (WIA), Adayar, Chennai, Tamil Nadu, India \\ ${ }^{3}$ Professor, Department of Radiation Oncology, Cancer Institute (WIA), Adayar, Chennai, Tamil Nadu, India \\ ${ }^{4}$ Senior Lecturer, Department of Oral Medicine and Radiology, Sri Ramachandra University, Chennai, Tamil Nadu, India \\ ${ }^{5}$ Professor, Department of Oral Medicine and Radiology, Sri Ramachandra University, Chennai, Tamil Nadu, India \\ ${ }^{6}$ Professor, Department of Pathology, Cancer Institute (WIA), Adayar, Chennai, Tamil Nadu, India \\ ${ }^{7}$ Professor, Department of Nuclear Medicine, Cancer Institute (WIA), Adayar, Chennai, Tamil Nadu, India \\ ${ }^{8}$ Associate Professor, Department of Surgical Oncology, Cancer Institute (WIA), Adayar, Chennai, Tamil Nadu, India
}

Correspondence: Arvind Krishnamurthy, Associate Professor, Department of Surgical Oncology, Division of Head and Neck Oncology, Cancer Institute (WIA), 36, Sardar Patel Road, Adayar, Chennai-20, Tamil Nadu, India, Phone: 9840448174 e-mail: drarvindkrishnamurthy @yahoo.co.in

\begin{abstract}
Hypertrophic osteoarthropathy $(\mathrm{HOA})$ is a condition characterized by periosteal reaction of tubular long bones and characteristic bulbous deformity of the digital tips with synovial effusion. It is usually encountered in conjunction with diseases involving the lungs, mediastinum, heart, pleura and gastrointestinal tract. The occurrence of HOA as a paraneoplastic manifestation in nasopharyngeal carcinoma is rare and only a handful of cases have been reported in literature and that too in the pediatric age group. An association with pulmonary metastasis was often described in these case reports. We present a case of HOA associated with nasopharyngeal carcinoma in a 20 years old gentleman in the absence of pulmonary metastasis.
\end{abstract}

Keywords: Hypertrophic osteoarthropathy, nasopharyngeal carcinoma, clubbing.

\section{INTRODUCTION}

Hypertrophic osteoarthropathy (HOA) is a condition characterized by periosteal reaction of tubular long bones and characteristic bulbous deformity of the digital tips with synovial effusion. ${ }^{1}$ It is usually encountered in conjunction with diseases involving the lungs, mediastinum, heart, liver, pleura and gastrointestinal tract. ${ }^{2,3}$ It is commonly seen as a paraneoplastic manifestation of thoracic malignancies. We present a case and discuss the evaluation and management of a 20 years old gentleman of nasopharyngeal cancer with HOA.

\section{CASE REPORT}

A 20 years old gentleman presented to us with a history of epistaxis and right sided neck swelling of 4 months duration. In addition he gave a history of right sided nasal obstruction and fullness in the right ear for the past 1 month. General examination revealed the classical symmetrical clubbing (Fig. 1) of recent onset (four months). Intraoral examination revealed moderate trismus. A nasopharyngeal endoscopic examination revealed predominantly a submucosal growth in the nasopharynx occluding the orifice of the right eustachian tube. A conglomerate node measuring $5 \times 4 \mathrm{~cm}$ was palpable in the right level II region of the neck. His neurological and skeletal examination was normal.

His hematological and biochemical parameters were normal except for an elevated serum alkaline phosphatase (531 IU/L). A computerized tomographic evaluation of head and neck revealed an enhancing mass lesion in the nasopharynx, right maxillary antrum, right sphenoid sinus, right parapharyngeal space and right infratemporal fossa with right upper deep cervical adenopathy.

After two noncontributory fine needle aspiration cytology attempts he underwent a right level two cervical lymph node biopsy which was suggestive of metastatic 


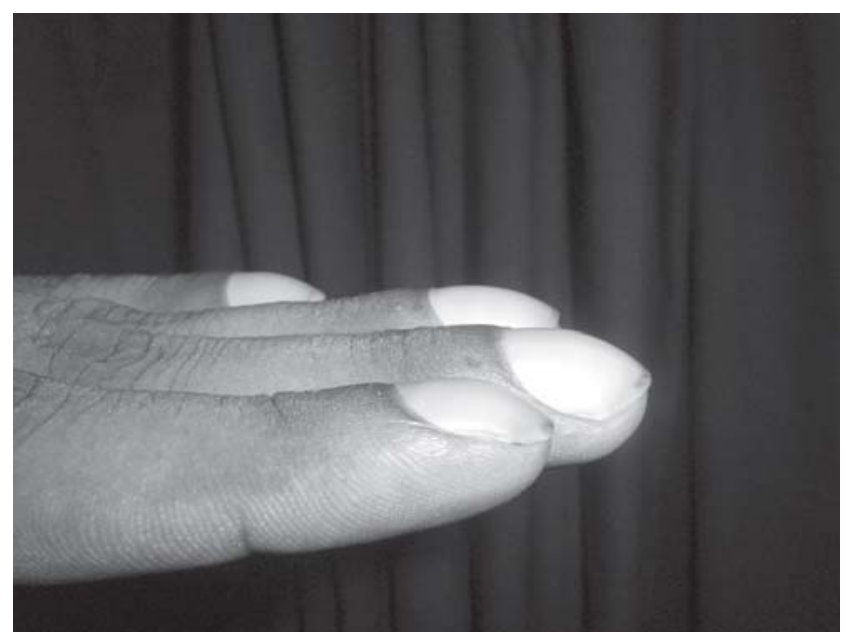

Fig. 1: Symmetrical clubbing

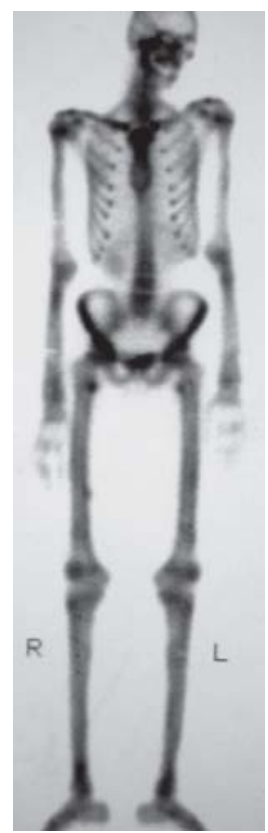

Fig. 2: Bone scan

carcinoma of nasopharyngeal type. His chest skiagram and ultrasound examination of the abdomen and pelvis was normal. He underwent a Technetium-99 MDP bone scan which showed intense bilateral symmetrical cortical uptake of radiopharmaceutical in all long bones (Tram line sign) (Fig. 2) without involving the axial skeleton. Skiagram of both lower limbs (Fig. 3) revealed bilateral symmetrical periosteal reaction confirming the bone scan findings of HOA. A diagnosis of HOA was made out based on the typical bone scintigraphic and radiological findings. A Positron emission tomography (PET-CT) revealed uptake in the nasopharynx and right level 2 node only confirming that this was indeed a locoregional disease (Fig. 4).

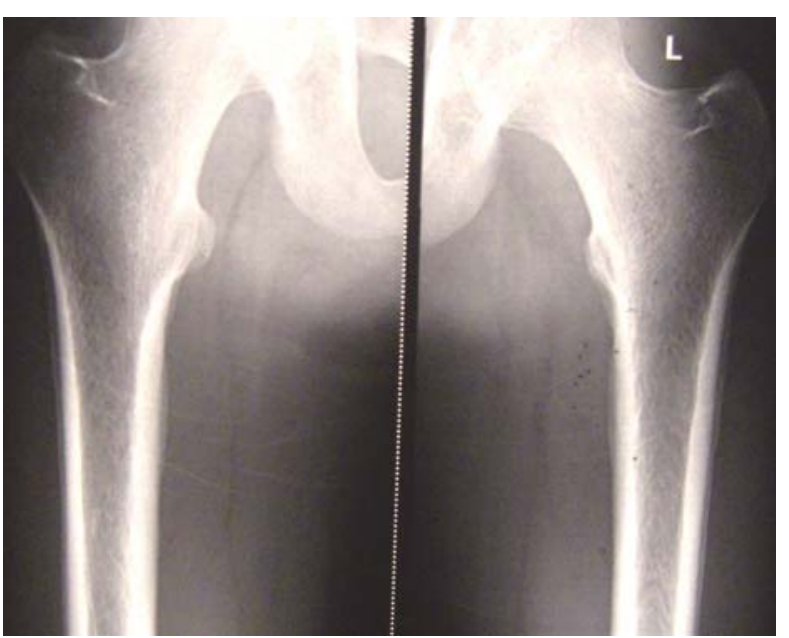

Fig. 3: Skiagram of the lower limbs

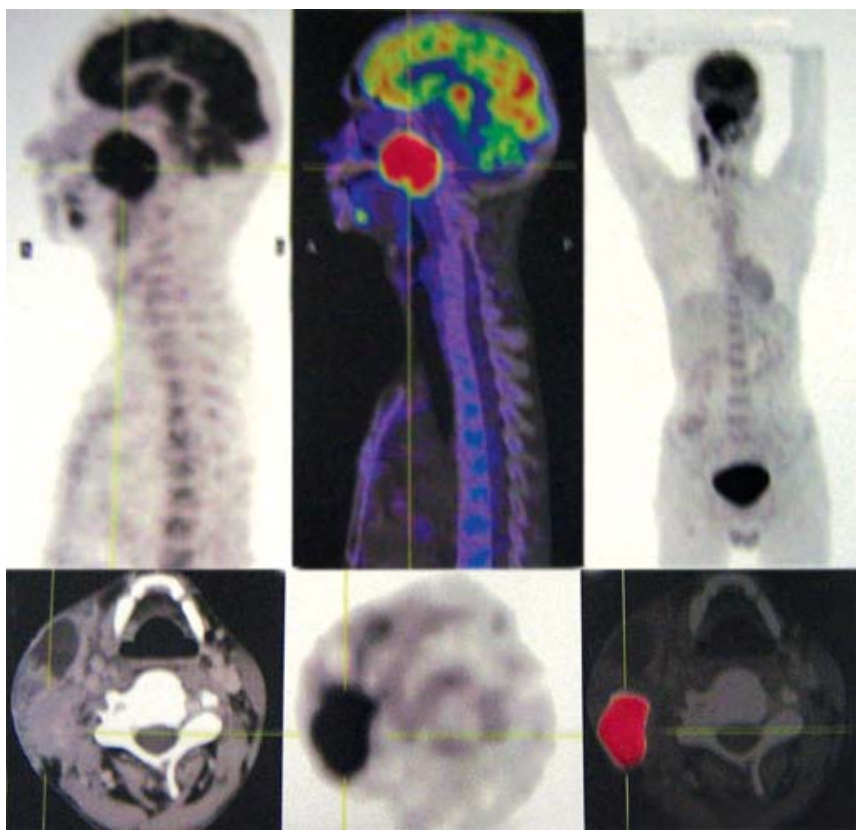

Fig. 4: PET-CT

The patient was thus staged as carcinoma nasopharynx T4 N1 M0 and was started on concurrent cisplatin and 5 Fluorouracil based chemotherapy and radiation. He is locoregionally controlled and is on regular follow-up for six months.

\section{DISCUSSION}

Hypertrophic osteoarthropathy (HOA) is a condition characterized by digital clubbing and periostosis of the long bones. ${ }^{4}$ Hippocrates first described digital clubbing more than 2500 years ago, hence the use of the term Hippocratic fingers. HOA can be primary or secondary. Primary HOA also known as pachydermoperiostosis and Touraine-Solente- 
Gole syndrome is a rare familial autosomal dominant condition ${ }^{4}$ while secondary HOA also called Pierre MarieBamberger syndrome is associated with pulmonary and nonpulmonary causes. Pulmonary causes include cystic fibrosis, pulmonary fibrosis, bronchiectasis, emphysema, pulmonary tuberculosis, primary and metastatic carcinomas of lung and mesothelioma. Nonpulmonary causes include diverse etiologies involving the cardiac, hepatic and gastrointestinal systems. It has also been described in diverse malignancies as well, non small cell lung carcinoma being the commonest. The other malignancies include metastatic phyllodes tumor of breast, epithelioid hemangioendothelioma, thymic, renal, gastric, pancreatic, esophageal carcinoma and rarely nasopharyngeal cancer with lung metastasis. ${ }^{5}$

The pathophysiology of HOA is still unclear; many different theories have been forwarded to explain the same, the popular two of them being the neurological theory of vagal stimulation ${ }^{6}$ and the humoral theory based on noninactivated platelet products in the venous circulation leading to HOA.

More than 260 cases of nasopharyngeal carcinoma associated with paraneoplastic syndrome have been reported in the literature, which include dermatomyositis, Cushing's syndrome, leukemoid reaction, sensory neuropathy and optic neuritis. ${ }^{7}$ The occurrence of HOA as a paraneoplastic manifestation in nasopharyngeal carcinoma is rare and only a handful of cases have been reported previously and that too in the pediatric age group. ${ }^{8}$

It is generally believed that the underlying disease usually appears prior to HOA. However, occasionally HOA precedes the onset of the disease; case reports have shown the development of clubbing and HOA in a patient with nasopharyngeal carcinoma six months prior to the radiological detection of pulmonary metastasis. ${ }^{8,9}$

Plain radiography and bone scans were the commonly used diagnostic tools in most of the described reports, the sensitivity of bone scans being better. CT and MRI scans were useful in elucidating the cause of HOA. However, neither radiographic results nor radionuclide findings are specific for HOA. We opted for a PET-CT mainly to rule out distant metastasis, especially bony and pulmonary; interestingly we found that HOA was not PET avid as against some cases where in a diffuse uptake was seen. ${ }^{10}$
The treatment of HOA is the elimination of the underlying condition. HOA usually regresses after control of the primary diseases but digital clubbing generally persists. ${ }^{7,8,11}$

The underlying disease process generally determines the prognosis of HOA. The life expectancy of patients with HOA is similar to that in persons without HOA. HOA when occurring in nasopharyngeal carcinomas is usually associated with pulmonary metastasis. The overall outlook of nasopharyngeal cancers with HOA is hence very poor. ${ }^{8}$

\section{CONCLUSION}

We wish to highlight the rare association of HOA as a paraneoplastic syndrome in nasopharyngeal carcinomas, an association which seems to adversely affect the prognosis of the disease adversity based on the sparse literature available. PET-CT was used as an important staging investigation; an option we feel is worth exploring.

\section{REFERENCES}

1. Mannel MC. Hypertrophic osteoarthopathy. In Klippel JH Dieppe PA (Eds). Rheumatology London: Mosby 1998;8(46): 1-4.

2. Aufses AH, Aufees BH. Hypertrophic osteoarthopathy in association with pulmonary metastases from extra thoracic malignancy. Dis Chest 1960;38:399-402.

3. Kaj CJ, Rossenhery MA. Burd and Hypertrophic Osteoarthopathy and childhood Hodgkin's disease. Radiology 1970; 112:177-78.

4. A Sreekumar. Hypertrophic pulmonary osteoarthropathy detected by Tc -99m MDP bone scintigraphy. Ind J Radiol Imag 2006;16(1):17-18.

5. Vera Sempere FJ, Botella Rod Riguez MS. Hypertrophic Osteoarthopathy in a patient with nasopharyngeal carcinoma. Acta Otorhinolaryngol Esp Jan-Feb 1995;46(1):67-70.

6. Martinez-Lavin M. Pathogenesis of hypertrophic osteoarthropathy. Clin Exp Rheumatol May-June 10 1992;Suppl 7.

7. Toroc, Rinaldo A, Politi M. Paraneoplastic syndrome in patient with nasopharyngeal cancer. Auris Nasus, Larnze Oct 2009;36(5):513-20. Epub 2008 Dec 27.

8. Biswal BM, Kareem A, Ahmed NM. Hypertrophic Osteroarthropathy: An unusual manifestation in nasopharyngeal cancer. Australas Radiol Feb 2001;45(1):71-73.

9. Gibbs DD, Schiller KFR, Stovin PGI: Lung metastases heralded by hypertrophic pulmonary osteoarthropathy. Lancet 1960; 1:623-25.

10. Hypertrophic pulmonary osteoarthropathy diagnosed by FDG PET-CT in a patient with lung adenocarcinoma Makis W, Abikhzer G, Rush C. Clin Nucl Med Sep 2009;34(9):625-27.

11. Kurzrock R, Cohen CR. Cutaneous Paraneoplastic Syndromes in solid tumors. Am J Med 1995;99:662-71. 